\title{
Overlapping Additive Schwarz preconditioners for isogeometric collocation discretizations of linear elasticity
}

\author{
D. Cho ${ }^{\mathrm{a}}$, L. F. Pavarino ${ }^{\mathrm{b}}, \mathrm{S} . \mathrm{Scacchi}^{\mathrm{c}}$ \\ ${ }^{a}$ Department of Mathematics, Dongguk University, Pil-dong 3-ga, Jung-gu, Seoul, 04620, South Korea. \\ ${ }^{b}$ Dipartimento di Matematica, Università degli Studi di Pavia, Via Ferrata 5, 27100 Pavia, Italy \\ ${ }^{c}$ Dipartimento di Matematica, Università degli Studi di Milano, Via Saldini 50, 20133 Milano, Italy
}

\begin{abstract}
Overlapping Additive Schwarz (OAS) preconditioners are here constructed for isogeometric collocation discretizations of the system of linear elasticity in both two and three space dimensions. Isogeometric collocation methods are recent variants of isogeometric analysis based on the numerical approximation of the strong form of partial differential equations at appropriate collocation points. Numerical results in two and three dimensions show that two-level OAS preconditioners are scalable in the number of subdomains $N$, quasi-optimal with respect to the mesh size $h$ and optimal with respect to the spline polynomial degree $p$. Moreover, two-level OAS preconditioners are more robust than one-level OAS and non-preconditioned GMRES solvers when the material tends to the incompressible limit, as well as in the presence of strong deformation of the NURBS geometry.
\end{abstract}

\section{Introduction}

Isogeometric analysis (IGA) is a numerical methodology, first introduced in [29], for the approximation of partial differential equations (PDEs). IGA is based on using Non-Uniform Rational B-Splines (NURBS), a standard in the Computer Aided Design (CAD) community, not only as basis functions representing CAD geometries, but also as the basis for the discrete solution space of PDEs.

Among the advantages of this approach with respect to the finite element method, we mention in particular the exact representation of CAD geometries and the greater control of the regularity of the discrete space. Indeed, spaces of global $C^{k}$ regularity can be easily implemented, yielding fewer degrees of freedom, better performance in case of vibrations, easier approximation of higher order problems and other advantages, see e.g. [22]. For a theoretical convergence analysis of IGA approximations of PDEs, we refer to [5] and the recent review [7]. Thanks to its flexibility, IGA has been successfully applied in diverse fields such as fluid dynamics [6, 28], structural mechanics [2], electromagnetics [16], computational electrocardiology [17], wave equations [53].

Assembling the stiffness matrices arising from isogeometric Galerkin approximations of PDEs might become computationally very expensive when the spline polynomial degree increases due to high-order quadrature rules. In order to speedup the stiffness matrices assembly, isogeometric collocation methods have been proposed in $[1,2,34]$. The main advantages of the collocation approach are that building the collocation stiffness matrix requires only one evaluation per basis

Email addresses: durkbin@dongguk.edu (D. Cho), luca.pavarino@unipv.it (L. F. Pavarino), simone.scacchi@unimi.it (S. Scacchi) 
function and the associated linear system turns out to be sparser than in the Galerkin case. We refer to [46] for a comparison of the computational cost of collocation and Galerkin IGA techniques.

The linear systems arising from IGA discretizations of elliptic problems can be very ill-conditioned when the spline polynomial degree $p$ increases and the development of efficient IGA solvers is a quite challenging issue in both Galerkin and collocation IGA. While research on domain decomposition solvers for IGA is a relatively recent field compared with the more established field of finite and spectral element solvers, some works have been proposed. Overlapping Additive Schwarz (OAS) preconditioners for Galerkin IGA approximations of scalar elliptic equations have been constructed and analyzed in [8], and then extended to linear elasticity and Stokes equations in [9], whereas OAS preconditioners for collocation IGA approximations of scalar elliptic equations have been introduced in [11]. Balancing Domain Decomposition by Constraints (BDDC) preconditioners for Galerkin IGA have been studied in $[10,13,14,43]$. In addition to our previous works, we also mention $[15,18,19]$ on BPX preconditioners, $[31,32,39]$ on IGA multigrid, $[35,36,37,38]$ on IGA Discontinuous Galerkin methods, and [40, 48, 49, 42] on other IGA solvers. A recent comparison between spectral elements and IGA discretizations and solvers can be found in [33].

The main novelty of the present work is to develop scalable OAS preconditioners for collocation IGA approximations of the linear elasticity system. This extends our previous works on standard Galerkin IGA discretizations for scalar elliptic problems [8], linear elasticity and Stokes systems [9], and on collocation IGA for scalar elliptic problems only [11]. OAS preconditioners are based on a geometric decomposition of the computational domain into overlapping subdomains. A onelevel OAS (OAS(1)) preconditioner is first constructed and it consists of solving concurrently a local collocation problem on each overlapping subdomain. The resulting algorithm is completely parallel. However, it is well known, see e.g. [47, 50], that the performance of one-level preconditioners deteriorates when the number of subdomains increases, because they are not scalable. Thus, we also propose a two-level preconditioner $(\mathrm{OAS}(2))$ which employs, in addition to the local problems, a coarse collocation problem. Since the isogeometric collocation system is non-symmetric, these OAS preconditioners are accelerated by the GMRES iterative method. In our numerical study, we first investigate how the GMRES iteration counts depend on the mesh size $h$ and number of subdomains $N$. We also study the behavior of the solvers in terms of the polynomial degree $p$, regularity index $k$, the Poisson ratio $\nu$, as well as in the presence of domain deformation. We find that two-level OAS preconditioners are scalable in $N$, quasi-optimal with respect to the IGA subdomain to mesh size ratio $H / h$ (i.e. with iteration counts which depend sublinearly on $H / h$ ) and optimal with respect to the IGA polynomial degree $p$ (i.e. with iteration counts which are independent of $p$ ). Moreover, two-level OAS preconditioners are more robust than one-level OAS and non-preconditioned GMRES solvers when the material tends to the incompressible limit, as well as in the presence of strong deformation of the NURBS geometry.

The present paper is organized as follows. In Section 2, we give a brief presentation of B-splines and NURBS. Section 3 introduces isogeometric collocation methods where the choice of collocation points is discussed. In Section 4, we present the proposed overlapping domain decomposition preconditioners. The results of several numerical tests in two and three dimensions are reported in Section 5 .

\section{B-splines and NURBS}

In this section, we describe briefly B-splines, NURBS, the basics of IGA and an introduction to the proposed discretization method. For more details on NURBS and IGA, we refer to [44, 45] and [29], respectively. 


\subsection{B-spline functions and spaces}

Univariate B-splines are scalar piecewise polynomials functions defined on $\mathbb{R}$, obtained as linear combinations of B-spline basis functions, introduced below. Denote as knot vector a set of nondecreasing real numbers representing coordinates in the parametric space, i.e. the unit interval,

$$
\left\{\xi_{1}=0, \ldots, \xi_{n+p+1}=1\right\},
$$

where $p$ is the polynomial degree of the $\mathrm{B}$-spline and $n$ is the number of basis functions (and control points) necessary to describe it. We call patch the interval $\left(\xi_{1}, \xi_{n+p+1}\right)$. The knot vector is denoted as uniform if its knots are uniformly-spaced and non-uniform otherwise. The maximal multiplicity of a knot is $p+1$. A knot vector is called open if its first and last knots have multiplicity $p+1$. In the following, we always employ open knot vectors. We recall that B-spline basis functions resulting from open knot vectors are interpolatory at the end points of the parametric interval $\widehat{I}:=(0,1)$, but in general they are not interpolatory at interior knots.

Given a knot vector, univariate B-spline basis functions are defined recursively. For $p=0$, i.e. the piecewise constants case, we set

$$
N_{i}^{0}(\xi)=\left\{\begin{array}{l}
1 \text { if } \xi_{i} \leq \xi<\xi_{i+1} \\
0 \text { otherwise }
\end{array}\right.
$$

Then, for $p \geq 1$ :

$$
N_{i}^{p}(\xi)= \begin{cases}\frac{\xi-\xi_{i}}{\xi_{i+p}-\xi_{i}} N_{i}^{p-1}(\xi)+\frac{\xi_{i+p+1}-\xi}{\xi_{i+p+1}-\xi_{i+1}} N_{i+1}^{p-1}(\xi) & \text { if } \xi_{i} \leq \xi<\xi_{i+p+1} \\ 0 & \text { otherwise }\end{cases}
$$

where we assume $\frac{0}{0}:=0$ in order to incorporate repeated knots. It follows that the generic basis function $N_{i}^{p}$ has support $\Theta_{i}:=\operatorname{supp}\left(N_{i}^{p}\right)=\left(\xi_{i}, \xi_{i+p+1}\right), \quad i=1,2, . ., n$. The B-spline basis functions are linearly independent and they form a partition of unity. Figure 1 shows an example consisting of $n=9$ cubic basis functions generated from the simple open knot vector $\boldsymbol{\xi}=\{0,0,0,0,1 / 6,1 / 3,1 / 2,2 / 3,5 / 6,1,1,1,1\}$.

If all interior knots are not repeated, thus if they have multiplicity 1, then the B-spline basis functions are $C^{p-1}$-continuous. If a knot has multiplicity $\alpha$, the basis is $C^{k}$-continuous, with $k=p-\alpha$, at that knot. Finally, the univariate B-spline space is defined by

$$
\widehat{\mathcal{S}}_{h}=\operatorname{span}\left\{N_{i}^{p}(\xi), i=1, \ldots, n\right\} .
$$

Multi-dimensional B-spline functions are easily constructed by tensor products. Here we consider the two-dimensional case for simplicity and the higher-dimensional case is analogous. Let $\widehat{\Omega}:=(0,1) \times(0,1)$ be the two-dimensional parametric space. Consider the knot vectors $\left\{\xi_{1}=\right.$ $\left.0, \ldots, \xi_{n+p+1}=1\right\}$ and $\left\{\eta_{1}=0, \ldots, \eta_{m+q+1}=1\right\}$, and a net of $n \times m$ control points $\mathbf{C}_{i, j}$. Univariate B-spline basis functions $N_{i}^{p}$ and $M_{j}^{q}$ (with $i=1, \ldots, n$ and $j=1, \ldots, m$ ) of degree $p$ and $q$, respectively, are defined from the knot vectors. The bivariate B-spline basis on $\widehat{\Omega}$ is then defined by tensor product as

$$
B_{i, j}^{p, q}(\xi, \eta)=N_{i}^{p}(\xi) M_{j}^{q}(\eta) .
$$

We remark that the two knot vectors $\left\{\xi_{1}=0, \ldots, \xi_{n+p+1}=1\right\}$ and $\left\{\eta_{1}=0, \ldots, \eta_{m+q+1}=1\right\}$ generate a mesh of rectangular elements in the parametric space. As done in (4), we can now define the bivariate B-spline space as

$$
\widehat{\mathcal{S}}_{h}=\operatorname{span}\left\{B_{i, j}^{p, q}(\xi, \eta), i=1, \ldots, n, j=1, \ldots, m\right\} .
$$




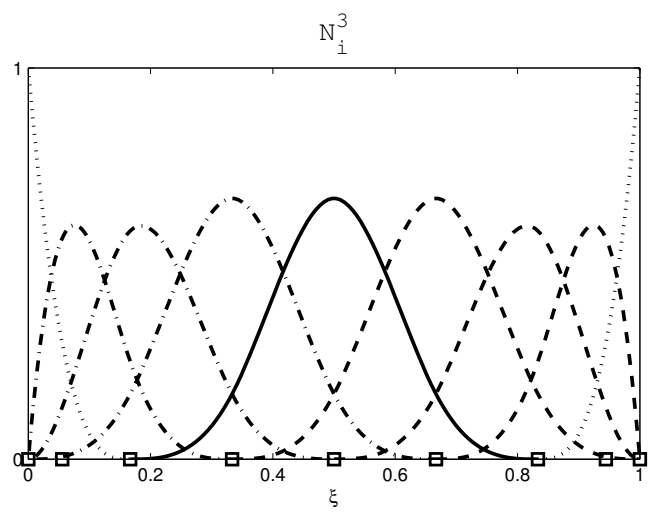

(a) $r=0$

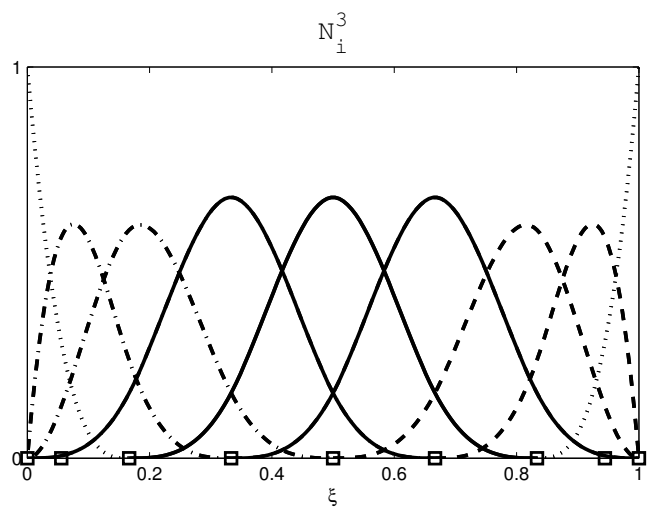

(b) $r=1$

Figure 1: Cubic basis functions formed from $\boldsymbol{\xi}=\{0,0,0,0,1 / 6,1 / 3,1 / 2,2 / 3,5 / 6,1,1,1,1\}$. For various $r$, $\widehat{V}_{1}$ is the span of basis functions drawn with dash-dot and solid lines and $\widehat{V}_{2}$ is the span of basis functions drawn with solid and dashed lines, in two subdomains $\widehat{I}_{1}=(0,1 / 2)$ and $\widehat{I}_{2}=(1 / 2,1)$ of $\widehat{I}$. In particular, the basis functions in common are those drawn with a solid line. The small rectangles on the x-axis denote the Greville abscissae associated with the knot vector $\boldsymbol{\xi}$.

\subsection{NURBS functions and spaces}

Univariate NURBS basis functions of degree $p$ are given by

$$
R_{i}^{p}(\xi)=\frac{N_{i}^{p}(\xi) \omega_{i}}{\sum_{\hat{\imath}=1}^{n} N_{\hat{\imath}}^{p}(\xi) \omega_{\hat{\imath}}}=\frac{N_{i}^{p}(\xi) \omega_{i}}{w(\xi)},
$$

where the denominator $w(\xi)=\sum_{\hat{1}=1}^{n} N_{\hat{1}}^{p}(\xi) \omega_{\hat{1}} \in \widehat{\mathcal{S}}_{h}$ is called the weight function.

A NURBS curve is then defined by

$$
\mathbf{C}(\xi)=\sum_{i=1}^{n} R_{i}^{p}(\xi) \mathbf{C}_{i}
$$

where $\mathbf{C}_{i} \in \mathbb{R}^{2}$ are control points.

Analogously to the B-spline case, NURBS basis functions on the two-dimensional parametric space $\widehat{\Omega}=(0,1) \times(0,1)$ are defined by

$$
R_{i, j}^{p, q}(\xi, \eta)=\frac{B_{i, j}^{p, q}(\xi, \eta) \omega_{i, j}}{\sum_{\hat{\imath}=1}^{n} \sum_{\hat{\jmath}=1}^{m} B_{\hat{1}, \hat{\jmath}}^{p, q}(\xi, \eta) \omega_{\hat{\imath}, \hat{\jmath}}}=\frac{B_{i, j}^{p, q}(\xi, \eta) \omega_{i, j}}{w(\xi, \eta)},
$$

where the denominator $w(\xi, \eta)$ is the weight function. The continuity and support of NURBS basis functions are the same as for B-splines and NURBS spaces are the span of the basis functions (8).

Given a single-patch domain $\Omega \subset \mathbb{R}^{2}$ associated with a net of $n \times m$ control points $\mathbf{C}_{i, j} \in \mathbb{R}^{2}$, we introduce the geometrical map $\mathbf{F}: \widehat{\Omega} \rightarrow \Omega$

$$
\mathbf{F}(\xi, \eta)=\sum_{i=1}^{n} \sum_{j=1}^{m} R_{i, j}^{p, q}(\xi, \eta) \mathbf{C}_{i, j}
$$

The space of NURBS scalar fields on the domain $\Omega$ is defined by the isoparametric approach as the span of the push-forward of the basis functions (8)

$$
\mathcal{N}_{h}:=\operatorname{span}\left\{R_{i, j}^{p, q} \circ \mathbf{F}^{-1}, \text { with } i=1, \ldots, n ; j=1, \ldots, m\right\} .
$$


The physical space elements are the images of the elements in the parametric space, therefore the physical mesh on $\Omega$ is

$$
\mathcal{T}_{h}=\left\{\mathbf{F}\left(\left(\xi_{i}, \xi_{i+1}\right) \times\left(\eta_{j}, \eta_{j+1}\right)\right), \text { with } i=1, \ldots, n+p, j=1, \ldots, m+q\right\},
$$

(the empty elements are not considered).

For further details and a complete presentation of NURBS functions, we refer the interested reader to the book by Farin [27].

\section{Isogeometric analysis and collocation methods}

\subsection{The linear elasticity problem}

We consider an elastic material body $\Omega \subset \mathbb{R}^{d}, d=2,3$, that is obtained by the NURBS geometrical map (9), that is, $\Omega=\mathbf{F}(\widehat{\Omega})$. Let $\mathbf{u}$ be the displacement field and $\mathbf{f}$ be the body force density. We consider the linear elastic deformation problem

$$
\operatorname{div} \mathbb{C} \varepsilon(\mathbf{u})+\mathbf{f}=0 \quad \text { in } \Omega
$$

Here, $\boldsymbol{\varepsilon}(\mathbf{u})$ is the symmetric gradient of $\mathbf{u}$ and the fourth order tensor $\mathbb{C}$ is defined by

$$
\mathbb{C} \boldsymbol{\tau}=2 \mu \boldsymbol{\tau}+\lambda \operatorname{tr}(\boldsymbol{\tau}) \mathbf{I}
$$

for all second order tensors $\boldsymbol{\tau}$, and $\operatorname{tr}(\boldsymbol{\tau})$ is the trace of $\boldsymbol{\tau} . \mu=\mu(x) \geq \mu_{0}>0$ is the shear modulus, $0 \leq \nu=\nu(x)<1 / 2$ the Poisson's ratio, and $\lambda=\frac{2 \mu \nu}{1-2 \nu}$.

Let $\Gamma_{D}$ and $\Gamma_{N}$ be two open subsets of $\partial \Omega$ such that $\partial \Omega=\bar{\Gamma}_{D} \cup \bar{\Gamma}_{N}$ and $\Gamma_{D} \cap \Gamma_{N}=\emptyset$. We suppose that the body is clamped on $\Gamma_{D}$ and it is subjected to a given traction $\mathbf{g}: \Gamma_{N} \rightarrow \mathbb{R}^{d}$. In other words, we impose the displacement boundary condition on $\Gamma_{D}$

$$
\mathbf{u}=0 \quad \text { on } \Gamma_{D},
$$

and the traction boundary condition on $\Gamma_{N}$

$$
\mathbb{C} \varepsilon(\mathbf{u}) \cdot \mathbf{n}=\mathbf{g} \quad \text { on } \Gamma_{N},
$$

where $\mathbf{n}$ is the unit outward normal at each point of the boundary.

\subsection{Isogeometric collocation methods}

We now define the isogeometric approximation spaces in $2 \mathrm{D}$, the $3 \mathrm{D}$ cases being analogous. For simplicity, we consider only a pure displacement problem, that is, $\Gamma_{D}=\partial \Omega$. As observed in our previous work [5], discrete space with homogeneous Dirichlet boundary conditions can be obtained by eliminating the first and last function in each coordinate. Hence, we define the spline space in parameter space $\widehat{\Omega}$ as

$$
\widehat{V}_{h}=\left[\widehat{\mathcal{S}}_{h} \cap H_{0}^{1}(\widehat{\Omega})\right]^{d}=\left[\operatorname{span}\left\{B_{i, j}^{p, q}(\xi, \eta), i=2, \ldots, n-1, j=2, \ldots, m-1\right\}\right]^{d},
$$

while the NURBS space in physical space $\Omega$ is defined as

$$
V_{h}=\left[\mathcal{N}_{h} \cap H_{0}^{1}(\Omega)\right]^{d}=\left[\operatorname{span}\left\{R_{i, j}^{p, q} \circ \mathbf{F}^{-1}, i=2, \ldots, n-1 ; j=2, \ldots, m-1\right\}\right]^{d} .
$$

Next, we recall the isogeometric collocation method for the pure displacement problem (12), see $[1,2,46]$ for more details. To simplify the presentation, we consider the case where the collocation 
points are chosen to be the Greville abscissae see [24]. The collocation points play a crucial role in the stability and good behavior of the discrete problem: the Demko abscissae [25] and Galerkin superconvergent points [41] can be alternatively selected as other collocation points. We show a comparison of these three choices of collocation points in the numerical results of Sec. 5, Table 2.

We denote by $\bar{\xi}_{i}, i=1, \ldots, n$, the Greville abscissae associated to the knot vector $\left\{\xi_{1}, \ldots, \xi_{n+p+1}\right\}$ :

$$
\bar{\xi}_{i} \doteq\left(\xi_{i+1}+\xi_{i+2}+\ldots+\xi_{i+p}\right) / p
$$

(see, e.g. Figure 1). Analogously, $\bar{\eta}_{j}, j=1, \ldots, m$, denote the Greville abscissae associated to the knot vector $\left\{\eta_{1}, \ldots, \eta_{m+q+1}\right\}$. By tensor product, we define the collocation points $\tau_{i j}$ in the physical domain $\bar{\Omega}$ :

$$
\tau_{i j}=\mathbf{F}\left(\widehat{\tau}_{i j}\right), \quad \widehat{\tau}_{i j}=\left(\bar{\xi}_{i}, \bar{\eta}_{j}\right) \in \overline{(\widehat{\Omega})},
$$

for $i=1, \ldots, n, j=1, \ldots, m$.

The isogeometric collocation problem with Greville abscissae reads:

$$
\begin{cases}\text { Find } \mathbf{u}_{h} \in V_{h} \text { such that: } & \\ \operatorname{div} \mathbb{C} \boldsymbol{\varepsilon}\left(\mathbf{u}_{h}\right)\left(\tau_{i j}\right)+\mathbf{f}\left(\tau_{i j}\right)=0 & i=2, \ldots, n-1, j=2, \ldots, m-1, \\ \mathbf{u}_{h}\left(\tau_{i j}\right)=0 & (i, j) \in(\{1, n\} \times\{1, \ldots, m\}) \cup(\{1, \ldots, n\} \times\{1, m\}) .\end{cases}
$$

Problems involving different boundary conditions can be dealt with similarly. Throughout the paper, we assume that the ensuing discrete spline and NURBS spaces are at least $C^{2}$ so that the equations in (16) are well defined. This means that cubic or higher degree polynomials are considered (i.e., $p \geq 3$ ) and the number of repetitions of any internal knot never exceeds $p-2$. Even in weaker regularity cases, IGA collocation methods can be easily modified (see [2] for details).

Many open issues remain in the mathematical theory of isogeometric collocation methods in higher dimensions, although several numerical tests in the literature show the stability and convergence of the method for many problems of practical interest.

\section{Overlapping Schwarz preconditioners}

We now introduce overlapping additive Schwarz (OAS) preconditioners for the IGA collocation problem (16).

\subsection{Subdomains and subspace decomposition}

The subdomains and subspace decomposition is built first for the space spanned by spline functions in parameter space and then is extended by the geometrical map to the NURBS space in the physical domain. We start in one spatial dimension and then extend the construction to higher dimensions using the tensor product structure. Given a knot vector $\left\{\xi_{1}=0, \ldots, \xi_{n+p+1}=1\right\}$, we select a subset $\left\{\xi_{i_{k}}: 1 \leq k \leq N+1\right\}$ of interface knots where $\xi_{i_{1}}=0, \xi_{i_{N+1}}=1$ and $\xi_{i_{k}} \leq \xi_{i_{k+1}}$. This subset induces a decomposition of the closure of the reference interval

$$
\overline{(\widehat{I})}=[0,1]=\overline{\left(\bigcup_{k=1, . ., N} \widehat{I}_{k}\right)}, \text { with } \widehat{I}_{k}=\left(\xi_{i_{k}}, \xi_{i_{k+1}}\right)
$$

which we assume to have similar characteristic diameters $H_{k}:=\operatorname{diam}\left(\widehat{I}_{k}\right) \approx H, 1 \leq k \leq N$. To each internal interface knot $\xi_{i_{k}}, 2 \leq k \leq N$, we associate an index $2 \leq s_{k} \leq N-1$ that satisfies both $s_{k}<i_{k}<s_{k}+p+1$ and $s_{k}<s_{k+1}$ (such index $s_{k}$ always exists; if it is not unique, any of the 
possible choices will work). The support of the associated basis function $N_{s_{k}}^{p}$ intersects both $\widehat{I}_{k-1}$ and $\widehat{I}_{k}$.

Let us define $r \in \mathbb{N}$ as an integer counting the basis functions shared by adjacent subdomains, which we call the overlap index in the following. We can then define both an overlapping domain decomposition of $\widehat{I}$ and an overlapping space decomposition of $\widehat{V}$ as follows. We introduce the index sets

$$
\Theta_{k}=\left\{j \in \mathbb{N}: s_{k}-r \leq j \leq s_{k+1}+r\right\} \quad k=1,2, . ., N,
$$

with $1 \leq j \leq s_{2}+r$ for $\Theta_{1}$ and $s_{N}-r \leq j \leq n$ for $\Theta_{N}$. Then we define the local spaces

$$
\widehat{V}_{k}=\operatorname{span}\left\{N_{j}^{p}(\xi): j \in \Theta_{k}\right\} \quad k=1,2, . ., N,
$$

with the analogous exception for $\widehat{V}_{1}$ and $\widehat{V}_{N}$ (see, e.g. Figure 1), and the overlapping subdomains $\widehat{I}_{k}^{\prime}=\bigcup_{N_{j}^{p} \in \widehat{V}_{k}} \operatorname{supp}\left(N_{j}^{p}\right)=\left(\xi_{s_{k}-r}, \xi_{s_{k+1}+r+p+1}\right)$, with the analogous exception for $\widehat{I}_{1}^{\prime}$ and $\widehat{I}_{N}^{\prime}$.

It is clear that these subspaces $\left\{\widehat{V}_{k}\right\}_{k=1}^{N}$ form an overlapping space decomposition of the spline space $\widehat{V}$ and the subdomains $\widehat{I}_{k}^{\prime}$ form an overlapping decomposition of the domain $\widehat{I}$. The number of basis functions in common (in the univariate case) among adjacent local subspaces is $2 r+1$. For instance, $r=0$ expresses the minimal overlap consisting of just one common basis function between neighboring local subspaces.

The subdomains $\widehat{I}_{k}$ determine a coarse mesh and the corresponding knot vector

$$
\boldsymbol{\xi}_{0}=\left\{\xi_{1}, \xi_{2}, \ldots, \xi_{p}, \xi_{i_{1}}, \xi_{i_{2}}, \xi_{i_{3}}, \ldots, \xi_{i_{N-1}}, \xi_{i_{N}}, \xi_{i_{N+1}}, \xi_{n+2} \ldots, \xi_{n+p+1}\right\},
$$

such that the distance between adjacent distinct knots is of the order $H, \xi_{1}=\cdots=\xi_{p}=\xi_{i_{1}}=0$ and $\xi_{i_{N+1}}=\xi_{n+2}=\cdots=\xi_{n+p+1}=1$. The associated coarse spline space is defined to be

$$
\widehat{V}_{0}:=\widehat{\mathcal{S}}_{H}=\operatorname{span}\left\{N_{i}^{0, p}(\xi), i=1, \ldots, N_{c}\right\} .
$$

$\widehat{V}_{0}$ is a subspace of $\widehat{\mathcal{S}}_{h}$ since it has the same degree $p$ as $\widehat{\mathcal{S}}_{h}$.

In two spatial dimension, we define subdomains and overlapping subdomains by tensor products as

$$
\widehat{I}_{k}=\left(\xi_{i_{k}}, \xi_{i_{k+1}}\right), \quad \widehat{I}_{l}=\left(\eta_{j_{l}}, \eta_{j_{l+1}}\right), \quad \widehat{\Omega}_{k l}=\widehat{I}_{k} \times \widehat{I}_{l} \quad 1 \leq k \leq N, 1 \leq l \leq M .
$$

In addition, taking the indices $\left\{s_{k}\right\}_{k=2}^{N}$ associated to $\left\{\xi_{i_{k}}\right\}_{k=2}^{N}$ and the analogous indices $\left\{\bar{s}_{l}\right\}_{l=2}^{M}$ associated to $\left\{\eta_{j_{l}}\right\}_{l=2}^{M}$, we define the local index sets

$$
\Theta_{k l}=\left\{(i, j) \in \mathbb{N}^{2}: s_{k}-r \leq i \leq s_{k+1}+r, \bar{s}_{l}-r \leq j \leq \bar{s}_{l+1}+r\right\}, 1 \leq k \leq N, 1 \leq l \leq M,
$$

which yield the local and coarse subspaces

$$
\begin{aligned}
\widehat{V}_{k l} & =\operatorname{span}\left\{B_{i, j}^{p, q}(\xi, \eta):(i, j) \in \Theta_{k l}\right\} \\
\widehat{V}_{0} & =\operatorname{span}\left\{B_{i, j}^{\circ, q}:{ }_{{ }^{\circ}}^{p, q}(\xi, \eta):=N_{i}^{0, p}(\xi) M_{j}^{0, q}(\eta), i=1, \ldots, N_{c}, j=1, \ldots, M_{c}\right\} .
\end{aligned}
$$

These subspaces can be extended by the push-forward to the NURBS space $V$ in the physical domain $\Omega$. Therefore the local subspaces and the coarse space are, up to the usual exceptions for the boundary subdomains,

$$
\begin{aligned}
V_{k l} & =\operatorname{span}\left\{R_{i, j}^{p, q} \circ \mathbf{F}^{-1}:(i, j) \in \Theta_{k l}\right\}, \\
V_{0} & =\operatorname{span}\left\{\stackrel{\circ}{R}_{i, j}^{p, q} \circ \mathbf{F}^{-1}:=\left(\frac{\stackrel{\circ}{B}_{i, j}^{p, q} \stackrel{\circ}{\omega}_{i, j}}{w}\right) \circ \mathbf{F}^{-1}, i=1, \ldots, N_{c}, j=1, \ldots, M_{c}\right\},
\end{aligned}
$$

where $w$ is the weight function, see (8). The image of the subdomains in parameter space are the subdomains in physical space

$$
\Omega_{k l}=\mathbf{F}\left(\widehat{\Omega}_{k l}\right) .
$$




\subsection{Matrix form of overlapping additive Schwarz preconditioners}

Still focusing on the 2D case for simplicity, we construct the preconditioned Schwarz operator as follows.

Local problems. For $k=1, . ., N$, and $l=1, . ., M$, let the local restriction matrices $R_{k l}: V \rightarrow V_{k l}$ be defined as the transpose of the natural embedding matrices $R_{k l}^{T}: V_{k l} \rightarrow V$ and let $A_{k l}$ be the square matrix associated to the local collocation problems:

$$
\begin{cases}\text { find } u_{h}^{k l} \in V_{k l} \text { such that } & \\ \operatorname{div} \mathbb{C} \varepsilon\left(\mathbf{u}_{h}^{k l}\right)\left(\tau_{i j}\right)+\mathbf{f}\left(\tau_{i j}\right)=0 & (i, j) \in \Theta_{k l}^{i}, \\ \mathbf{u}_{h}^{k l}\left(\tau_{i j}\right)=0 & (i, j) \in \Theta_{k l}^{\partial},\end{cases}
$$

where

$$
\begin{aligned}
& \Theta_{k l}^{i}=\left\{(i, j) \in \Theta_{k l}:(i, j) \notin(\{1, n\} \times\{1, \ldots, m\}) \cup(\{1, \ldots, n\} \times\{1, m\})\right\} \\
& \Theta_{k l}^{\partial}=\Theta_{k l} \backslash \Theta_{k l}^{i},
\end{aligned}
$$

are the internal and boundary index sets. If $A$ is the global collocation matrix associated to the original problem (16), it is easy to see that the local matrices coincide with

$$
A_{k l}=R_{k l} A R_{k l}^{T}
$$

Coarse problem. In the analogy to (19), we consider a nested coarse space defining the coarse matrix by

$$
A_{0}=R_{0} A R_{0}^{T}
$$

where $R_{0}^{T}$ is the interpolation matrix from the coarse space $V_{0}$ to the fine NURBS space $V$. This coarse matrix corresponds to a collocation problem where each equation in the linear system can be viewed as a weighted sum of the collocation problem of the differential equation on more than one point. Different kinds of coarse problems could be used as well, see e.g. [11] for the scalar elliptic case.

The proposed Overlapping Additive Schwarz (OAS) operator can be written in matrix form as

$$
\mathbf{T}_{O A S}=\mathbf{B}_{O A S} A
$$

where the OAS preconditioner $\mathbf{B}_{O A S}$ can be the one-level version if we consider only the local problems or the two-level version if we consider both local and coarse problems:

$$
\begin{gathered}
\mathbf{B}_{O A S}=\mathbf{B}_{O A S(1)}=\sum_{k=1}^{N} \sum_{l=1}^{M} R_{k l}^{T} A_{k l}^{-1} R_{k l} \\
\mathbf{B}_{O A S}=\mathbf{B}_{O A S(2)}=R_{0}^{T} A_{0}^{-1} R_{0}+\sum_{k=1}^{N} \sum_{l=1}^{M} R_{k l}^{T} A_{k l}^{-1} R_{k l},
\end{gathered}
$$

The higher-dimensional case is analogous. An advantage of collocation IGA is that the assembly of the matrices $A_{k l}, A_{0}$ and $A$ is much cheaper than in Galerkin IGA (see [46]), since the collocation matrices are sparser than their Galerkin counterparts.

Other types of preconditioners (for instance, general multiplicative, hybrid ones and projectionlike operators $\widetilde{\mathbf{T}}_{k l}$ associated with inexact local solvers) could be used (see [47, 50] for further details), and will need future research. We can also see this preconditioning process as replacing the discrete system $A \mathbf{u}=\mathbf{f}$ with the preconditioned system

$$
\mathbf{T}_{O A S} \mathbf{u}=\mathbf{g}
$$

which can be solved by a Krylov subspace method with right-hand side $\mathbf{g}=\mathbf{B}_{O A S} \mathbf{f}$. 
We remark that, although the original problem is self-adjoint, the global matrix $A$ and the preconditioned operator are in general non-symmetric. Therefore, we use the GMRES iterative method instead of the conjugate gradient method in the solution process.

The numerical results reported in the next section indicate that our OAS solver for IGA collocation has scalability and convergence rates analogous to its IGA Galerkin counterpart. Therefore, we conjecture that an analogous convergence rate bound holds:

Conjecture. The GMRES iteration counts to solve the OAS preconditioned system (22) up to a given tolerance for the relative residual is bounded by

$$
\text { iter }_{O A S} \leq C g\left(\frac{H}{r h}\right)
$$

where $g$ is a sublinear function, $C$ is a constant independent of $N, h, H, r$ but not of $p, k$. We are currently unable to carry out a theoretical analysis of this conjecture since spectral bounds of the IGA collocation operators, as well as their basic approximation properties, are still open problems in the IGA collocation case.

\section{Numerical results}

The linear elasticity systems arising from the IGA collocation discretization are solved by the GMRES method with the 1-level (OAS(1)) and 2-level (OAS(2)) overlapping additive Schwarz preconditioners defined in (21), starting from a zero initial guess and a $10^{-6}$ reduction of the relative residual as stopping criterion. Our Matlab code is based on the isogeometric library GeoPDEs $[23,52]$ and all numerical tests have been run on a Linux workstation.

We investigate numerically the OAS GMRES convergence rate (i.e. the GMRES iteration counts with the OAS preconditioner) for two- and three-dimensional linear elasticity problems with respect to the following parameters:

- the isogeometric mesh size $h$;

- the spline polynomial degree $p$;

- the global spline regularity $k$;

- the number of subdomains $N$;

- the subdomain characteristic size $H$;

- the overlap index $r$.

We consider three numerical tests to study the numerical performance of our preconditioner for linear elasticity problems on various domains $\Omega \subset \mathbb{R}^{2}$, described below and we then consider a three-dimensional scalability test.

- (Parametric domain) The geometry and the solution are given by

$$
\begin{aligned}
& \Omega=(0,1)^{2} \subset \mathbb{R}^{2}, \quad \Gamma_{D}=\partial \Omega, \\
& \mathbf{u}=(\sin (2 \pi x) \sin (2 \pi y) \quad \sin (2 \pi x) \sin (2 \pi y))^{t}
\end{aligned}
$$

- (Quarter-ring domain) A non-homogeneous Dirichlet boundary value problem of linear elasticity has the exact solution

$$
\mathbf{u}=\left(\frac{1}{3 E}\left((1-\nu) r+\frac{4(1+\nu)}{r}\right) \cos \theta \quad \frac{1}{3 E}\left((1-\nu) r+\frac{4(1+\nu)}{r}\right) \sin \theta\right)^{t}
$$


where $(r, \theta)$ is the polar coordinating system in the quarter-ring domain $\Omega:=\left\{\left(x_{1}, x_{2}\right): 1<\right.$ $\left.x_{1}^{2}+x_{2}^{2}<4, x_{1}>0, x_{2}>0\right\}$ with $\Gamma_{D}=\partial \Omega$.

- (Curved domains) We take the exact solutions u subject to the body force $\mathbf{f}=\left(\begin{array}{ll}0 & 1\end{array}\right)^{t}$ on the boomerang-shaped domains presented in Figure 3 with $\Gamma_{D}=\partial \Omega$.

\subsection{Test 1: 2D weak scalability test}

We start performing a weak scalability test on the unit square, by increasing the number of subdomains $N$ and refining the mesh size $h$, while keeping fixed the ratio $H / h=8$, thus the same computational load per subdomain. We consider Greville collocation points, spline parameters $p=3, k=2$ and $p=4, k=3$, and overlap index $r=0$. The results are reported in Table 1 .

We first observe that the IGA collocation method, in terms of the $L^{\infty}$ norm, converges with the expected rate (see [1]), that is 2 for $p=3, k=2$ and 4 for $p=4, k=3$. Only the $\operatorname{OAS}(2)$ preconditioner exhibits a scalable behavior, because the GMRES iteration counts remain bounded from above independently of $N$, while $\operatorname{OAS}(1)$ iteration counts increase with $N$.

A fair performance comparison between $\mathrm{OAS}(1)$ and $\mathrm{OAS}(2)$ preconditioners should be done by means of parallel computations. However, our Matlab implementation is serial. Therefore, in order to mimick a parallel computation, we divide the CPU times needed for the solution of the local problems, which is the parallel part of the algorithm, by the number of subdomains. The results reported in Table 1 show that the CPU times of the OAS(2) preconditioner remain bounded when $N$ increases, while the CPU times of $\mathrm{OAS}(1)$ preconditioner increase significantly, because the iterations are not scalable.

\subsection{Test 2: 2D scalability in $N$ and quasi-optimality in $H / h$}

Table 2 reports the GMRES iteration counts of the $\operatorname{OAS}(1)$ and $\operatorname{OAS}(2)$ preconditioners on the unit square, where three types of collocation points (Greville, Demko and superconvergent) are taken into account, with $p=3, k=2$ and two overlap indices $(r=0$ and $r=1)$. Tables 3 and 4 display additional results for $p=4, k=3$ (square domain) and $p=3, k=2$ (quarter-ring domain), respectively. The results show the scalability of the $\mathrm{OAS}(2)$ preconditioner, because when $N$ is increasing while keeping fixed the ratio $H / h$ (moving along the diagonal of the tables), the iteration counts remain bounded from above by a constant independent of $N$; see also Figure 2 . On the other hand, the OAS(1) preconditioner is not scalable, because the iteration counts grow with $N$ (again moving along the diagonals of the tables). We also observe that moving instead along the tables rows (i.e. varying the ratio $H / h$ for fixed $N$ ), a less than linear dependence of the $\operatorname{OAS}(2)$ preconditioner on the ratio $H / h$; see also the plots in Figure 2.

\subsection{Test 3: solver performance when $\nu \rightarrow 0.5$}

Next, we consider the behavior of the non-preconditioned scheme and two OAS preconditioners for the almost incompressible elasticity system with the Poisson ratio $\nu$ increasing toward 0.5 and and fixed Young modulus $E=1 e+6$. The system on the quarter ring is discretized with $N=4 \times 4$ subdomains, ratio $H / h=8$ and minimal overlap $r=0$. Here we use the spline discretization parameters $p=3, k=2$. As expected in the primal formulation, the test results presented in Table 5 deteriorate for all the three methods, even though $\mathrm{OAS}(2)$ preconditioner has a better performance. It is well-known that a mixed reformulation of the elasticity system would be a good remedy for the locking phenomenon (such a study is beyond the scope of the present paper). 


\begin{tabular}{|c|c|c|c|c|c|c|c|}
\hline \multicolumn{8}{|c|}{$p=3, k=2$, unit square domain } \\
\hline \multirow[b]{2}{*}{$N$} & \multirow[b]{2}{*}{$1 / h$} & \multirow[b]{2}{*}{ err } & \multirow[b]{2}{*}{ rate } & \multicolumn{2}{|c|}{$\mathrm{OAS}(1)$} & \multicolumn{2}{|c|}{$\mathrm{OAS}(2)$} \\
\hline & & & & it. & time & it. & time \\
\hline $2 \times 2$ & 16 & $1.54 \mathrm{e}-2$ & - & 12 & $1.75 \mathrm{e}-2$ & 11 & $1.75 \mathrm{e}-2$ \\
\hline $4 \times 4$ & 32 & $3.90 \mathrm{e}-3$ & 1.98 & 27 & $2.19 \mathrm{e}-2$ & 16 & $1.31 \mathrm{e}-2$ \\
\hline $6 \times 6$ & 48 & $1.70 \mathrm{e}-3$ & 2.05 & 38 & $3.83 \mathrm{e}-2$ & 16 & $1.78 \mathrm{e}-2$ \\
\hline $8 \times 8$ & 64 & $9.74 \mathrm{e}-4$ & 1.94 & 48 & $3.16 \mathrm{e}-2$ & 16 & $1.23 \mathrm{e}-2$ \\
\hline $10 \times 10$ & 80 & $6.24 \mathrm{e}-4$ & 2.00 & 59 & $5.20 \mathrm{e}-2$ & 15 & $1.54 \mathrm{e}-2$ \\
\hline $12 \times 12$ & 96 & $4.33 \mathrm{e}-4$ & 2.00 & 70 & $5.90 \mathrm{e}-2$ & 14 & $1.38 \mathrm{e}-2$ \\
\hline $14 \times 14$ & 112 & $3.18 \mathrm{e}-4$ & 2.00 & 82 & $7.17 \mathrm{e}-2$ & 13 & $1.32 \mathrm{e}-2$ \\
\hline $16 \times 16$ & 128 & $2.44 \mathrm{e}-4$ & 1.98 & 93 & $4.97 \mathrm{e}-2$ & 13 & $8.10 \mathrm{e}-3$ \\
\hline \multicolumn{8}{|c|}{$p=4, k=3$, unit square domain } \\
\hline & & & & & $\overline{\mathrm{AS}}(1)$ & ( & $\mathrm{AS}(2)$ \\
\hline$N$ & $1 / h$ & err & rate & it. & time & it. & time \\
\hline $2 \times 2$ & 16 & $1.04 \mathrm{e}-4$ & - & 12 & $5.00 \mathrm{e}-2$ & 10 & $4.50 \mathrm{e}-2$ \\
\hline $4 \times 4$ & 32 & $6.92 \mathrm{e}-6$ & 3.91 & 21 & $5.81 \mathrm{e}-2$ & 14 & $4.19 \mathrm{e}-2$ \\
\hline $6 \times 6$ & 48 & $1.39 \mathrm{e}-6$ & 3.96 & 30 & $1.09 \mathrm{e}-1$ & 14 & $5.39 \mathrm{e}-2$ \\
\hline $8 \times 8$ & 64 & $4.46 \mathrm{e}-7$ & 3.95 & 38 & $9.30 \mathrm{e}-2$ & 13 & $3.39 \mathrm{e}-2$ \\
\hline $10 \times 10$ & 80 & $1.84 \mathrm{e}-7$ & 3.97 & 47 & $1.17 \mathrm{e}-1$ & 13 & $3.64 \mathrm{e}-2$ \\
\hline $12 \times 12$ & 96 & $8.90 \mathrm{e}-8$ & 3.98 & 55 & $2.02 \mathrm{e}-1$ & 12 & $5.01 \mathrm{e}-2$ \\
\hline $14 \times 14$ & 112 & $4.82 \mathrm{e}-8$ & 3.98 & 64 & $1.95 \mathrm{e}-1$ & 12 & $4.19 \mathrm{e}-2$ \\
\hline $16 \times 16$ & 128 & $2.83 \mathrm{e}-8$ & 3.99 & 73 & $1.60 \mathrm{e}-1$ & 11 & $2.85 \mathrm{e}-2$ \\
\hline
\end{tabular}

Table 1: Test 1, 2D weak scalability on the unit square, Greville collocation points. Error in $L^{\infty}$ norm with respect to exact solution (err), convergence rate (rate), GMRES iteration counts (it.) and CPU times in seconds (time), varying the number of subdomains $N$ and mesh size $1 / h$, while keeping fixed the ratio $H / h=8$, the overlap parameter $r=0$, $E=1, \nu=0.3$.
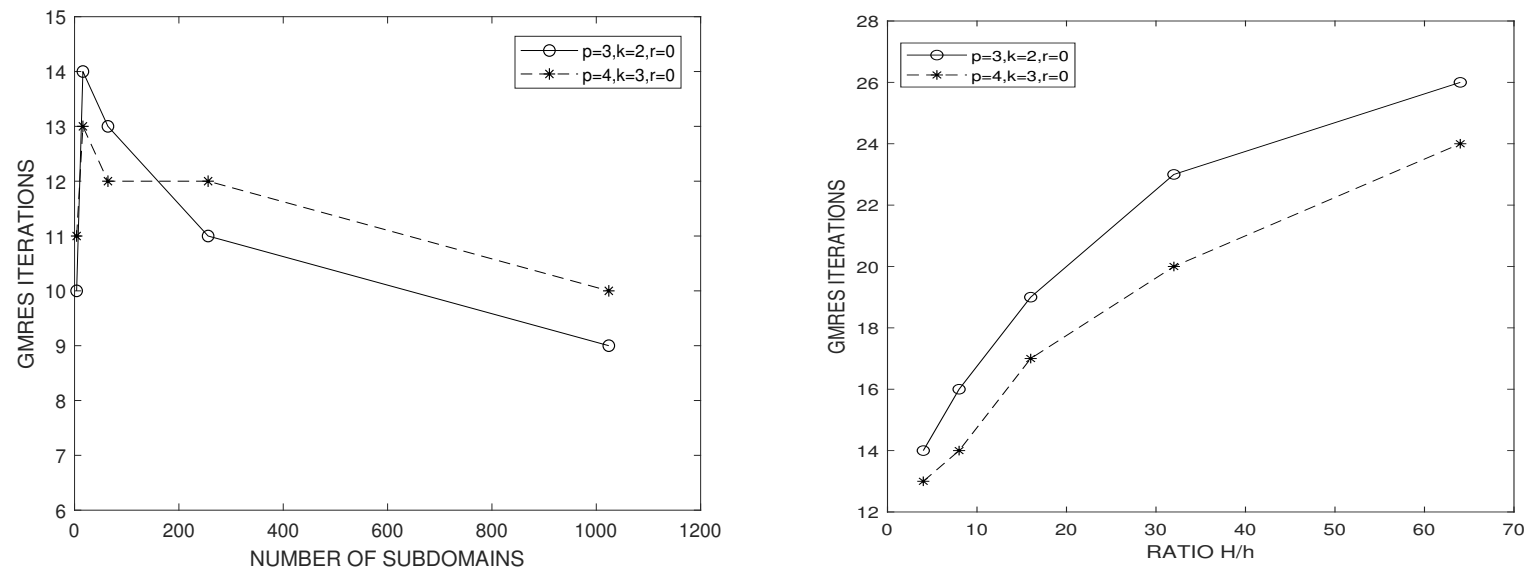

Figure 2: Test 2, 2D scalability in $N$ and quasi-optimality in $H / h$. 2-level OAS preconditioners with overlap $r=0$ $(p=3, k=2$ and $p=4, k=3)$ spline spaces on the unit square domain, Greville collocation points. GMRES iteration counts for increasing number of subdomains $N$ and fixed $H / h=4$ (left panel), and for increasing ratio $H / h$ and fixed $N=4 \times 4$ (right panel). 


\begin{tabular}{|c|c|c|c|c|c|c|c|c|c|c|c|c|}
\hline \multicolumn{13}{|c|}{$p=3, k=2$, unit square domain } \\
\hline \multicolumn{13}{|c|}{ OAS(1) - Greville collocation points } \\
\hline & \multicolumn{2}{|c|}{$1 / h=8$} & \multicolumn{2}{|c|}{$1 / h=16$} & \multicolumn{2}{|c|}{$1 / h=32$} & \multicolumn{2}{|c|}{$1 / h=64$} & \multicolumn{2}{|c|}{$1 / h=128$} & \multicolumn{2}{|c|}{$1 / h=256$} \\
\hline$N$ & $r=0$ & $r=1$ & $r=0$ & $r=1$ & $r=0$ & $r=1$ & $r=0$ & $r=1$ & $r=0$ & $r=1$ & $r=0$ & $r=1$ \\
\hline $2 \times 2$ & 10 & 9 & 12 & 11 & 15 & 13 & 18 & 16 & 20 & 18 & 16 & 21 \\
\hline $4 \times 4$ & - & - & 19 & 14 & 27 & 19 & 36 & 27 & 48 & 37 & 65 & 48 \\
\hline $8 \times 8$ & - & - & - & - & 34 & 22 & 48 & 34 & 67 & 49 & 93 & 69 \\
\hline $16 \times 16$ & - & - & - & - & - & - & 65 & 42 & 93 & 65 & 168 & 95 \\
\hline $32 \times 32$ & - & - & - & - & - & - & - & - & 159 & 83 & 259 & 164 \\
\hline $64 \times 64$ & - & - & - & - & - & - & - & - & - & - & 417 & 236 \\
\hline \multicolumn{13}{|c|}{ OAS(2) - Greville collocation points } \\
\hline & \multicolumn{2}{|c|}{$1 / h=8$} & \multicolumn{2}{|c|}{$1 / h=16$} & \multicolumn{2}{|c|}{$1 / h=32$} & \multicolumn{2}{|c|}{$1 / h=64$} & \multicolumn{2}{|c|}{$1 / h=128$} & \multicolumn{2}{|c|}{$1 / h=256$} \\
\hline$N$ & $r=0$ & $r=1$ & $r=0$ & $r=1$ & $r=0$ & $r=1$ & $r=0$ & $r=1$ & $r=0$ & $r=1$ & $r=0$ & $r=1$ \\
\hline $2 \times 2$ & 10 & 11 & 11 & 11 & 13 & 12 & 15 & 14 & 16 & 16 & 18 & 18 \\
\hline $4 \times 4$ & - & - & 14 & 13 & 16 & 14 & 19 & 16 & 23 & 19 & 26 & 23 \\
\hline $8 \times 8$ & - & - & - & - & 13 & 13 & 16 & 13 & 18 & 15 & 21 & 18 \\
\hline $16 \times 16$ & - & - & - & - & - & - & 11 & 12 & 13 & 11 & 15 & 13 \\
\hline $32 \times 32$ & - & - & - & - & - & - & - & - & 9 & 11 & 10 & 9 \\
\hline $64 \times 64$ & - & - & - & - & - & - & - & - & - & - & 8 & 9 \\
\hline
\end{tabular}

OAS(1) - Demko collocation points

\begin{tabular}{|c|c|c|c|c|c|c|c|c|c|c|c|c|}
\hline \multirow[b]{2}{*}{$N$} & \multicolumn{2}{|c|}{$1 / h=8$} & \multicolumn{2}{|c|}{$1 / h=16$} & \multicolumn{2}{|c|}{$1 / h=32$} & \multicolumn{2}{|c|}{$1 / h=64$} & \multicolumn{2}{|c|}{$1 / h=128$} & \multicolumn{2}{|c|}{$1 / h=256$} \\
\hline & $r=0$ & $r=1$ & $r=0$ & $r=1$ & $r=0$ & $r=1$ & $r=0$ & $r=1$ & $r=0$ & $r=1$ & $r=0$ & $r=1$ \\
\hline $2 \times 2$ & 10 & 9 & 12 & 11 & 15 & 13 & 18 & 16 & 20 & 18 & 16 & 21 \\
\hline $4 \times 4$ & - & - & 19 & 14 & 27 & 19 & 36 & 27 & 48 & 37 & 65 & 48 \\
\hline $8 \times 8$ & - & - & - & - & 34 & 23 & 48 & 34 & 67 & 49 & 93 & 69 \\
\hline $16 \times 16$ & - & - & - & - & - & - & 65 & 43 & 93 & 65 & 168 & 95 \\
\hline $32 \times 32$ & - & - & - & - & - & - & - & - & 159 & 85 & 259 & 164 \\
\hline $64 \times 64$ & - & - & - & - & - & - & - & - & - & - & 418 & 240 \\
\hline \multicolumn{13}{|c|}{ OAS $(2)$ - Demko collocation points } \\
\hline & \multicolumn{2}{|c|}{$1 / h=8$} & \multicolumn{2}{|c|}{$1 / h=16$} & \multicolumn{2}{|c|}{$1 / h=32$} & \multicolumn{2}{|c|}{$1 / h=64$} & \multicolumn{2}{|c|}{$1 / h=128$} & \multicolumn{2}{|c|}{$1 / h=256$} \\
\hline$N$ & $r=0$ & $r=1$ & $r=0$ & $r=1$ & $r=0$ & $r=1$ & $r=0$ & $r=1$ & $r=0$ & $r=1$ & $r=0$ & $r=1$ \\
\hline $2 \times 2$ & 10 & 11 & 11 & 11 & 13 & 12 & 15 & 14 & 16 & 16 & 18 & 18 \\
\hline $4 \times 4$ & - & - & 14 & 13 & 16 & 14 & 19 & 16 & 23 & 19 & 26 & 23 \\
\hline $8 \times 8$ & - & - & - & - & 13 & 13 & 16 & 13 & 18 & 15 & 21 & 18 \\
\hline $16 \times 16$ & - & - & - & - & - & - & 11 & 12 & 13 & 11 & 15 & 13 \\
\hline $32 \times 32$ & - & - & - & - & - & - & - & - & 9 & 11 & 10 & 9 \\
\hline $64 \times 64$ & - & - & - & - & - & - & - & - & - & - & 8 & 9 \\
\hline
\end{tabular}

OAS(1) - Superconvergent collocation points

\begin{tabular}{c|cc|cc|cc|cc|cc|cc}
\hline & \multicolumn{2}{|c|}{$1 / h=8$} & \multicolumn{2}{c|}{$1 / h=16$} & \multicolumn{2}{c|}{$1 / h=32$} & \multicolumn{2}{c|}{$1 / h=64$} & \multicolumn{2}{c}{$1 / h=128$} & \multicolumn{2}{c}{$1 / h=256$} \\
$N$ & $r=0$ & $r=1$ & $r=0$ & $r=1$ & $r=0$ & $r=1$ & $r=0$ & $r=1$ & $r=0$ & $r=1$ & $r=0$ & $r=1$ \\
\hline $2 \times 2$ & 12 & 10 & 15 & 12 & 18 & 15 & 20 & 18 & 23 & 20 & 22 & 23 \\
$4 \times 4$ & - & - & 21 & 17 & 29 & 21 & 39 & 29 & 51 & 39 & 63 & 49 \\
$8 \times 8$ & - & - & - & - & 39 & 27 & 54 & 38 & 72 & 53 & 94 & 71 \\
$16 \times 16$ & - & - & - & - & - & - & 74 & 51 & 106 & 72 & 144 & 101 \\
$32 \times 32$ & - & - & - & - & - & - & - & - & 149 & 102 & 248 & 146 \\
$64 \times 64$ & - & - & - & - & - & - & - & - & - & - & 386 & 278 \\
\hline
\end{tabular}

$\mathrm{OAS}(2)$ - Superconvergent collocation points

\begin{tabular}{c|cc|cc|cc|cc|cc|cc}
\hline & \multicolumn{2}{|c|}{$1 / h=8$} & \multicolumn{2}{c|}{$1 / h=16$} & \multicolumn{2}{c|}{$1 / h=32$} & \multicolumn{2}{c|}{$1 / h=64$} & \multicolumn{2}{c|}{$1 / h=128$} & \multicolumn{2}{c}{$1 / h=256$} \\
$N$ & $r=0$ & $r=1$ & $r=0$ & $r=1$ & $r=0$ & $r=1$ & $r=0$ & $r=1$ & $r=0$ & $r=1$ & $r=0$ & $r=1$ \\
\hline $2 \times 2$ & 11 & 13 & 12 & 12 & 14 & 12 & 16 & 15 & 18 & 17 & 19 & 18 \\
$4 \times 4$ & - & - & 15 & 14 & 16 & 14 & 19 & 16 & 22 & 19 & 27 & 23 \\
$8 \times 8$ & - & - & - & - & 15 & 16 & 16 & 13 & 19 & 15 & 21 & 18 \\
$16 \times 16$ & - & - & - & - & - & - & 13 & 16 & 13 & 12 & 15 & 13 \\
$32 \times 32$ & - & - & - & - & - & - & - & - & 12 & 15 & 11 & 11 \\
$64 \times 64$ & - & - & - & - & - & - & - & - & - & - & 10 & 13 \\
\hline
\end{tabular}

Table 2: Test 2, 2D scalability in $N$ and quasi-optimality in $H / h$. OAS preconditioners on the unit square domain, Greville, Demko and superconvergent collocation points. GMRES iteration counts varying the number of subdomains $N$, mesh size $1 / h$, overlap parameter $r$, for fixed $p=3, k=2, E=1 e+6, \nu=0.3$. 


\begin{tabular}{|c|c|c|c|c|c|c|c|c|c|c|c|c|}
\hline \multicolumn{13}{|c|}{$p=4, k=3$, unit square domain } \\
\hline \multicolumn{13}{|c|}{ OAS(1) - Greville collocation points } \\
\hline & \multicolumn{2}{|c|}{$1 / h=8$} & \multicolumn{2}{|c|}{$1 / h=16$} & \multicolumn{2}{|c|}{$1 / h=32$} & \multicolumn{2}{|c|}{$1 / h=64$} & \multicolumn{2}{|c|}{$1 / h=128$} & \multicolumn{2}{|c|}{$1 / h=256$} \\
\hline$N$ & $r=0$ & $r=1$ & $r=0$ & $r=1$ & $r=0$ & $r=1$ & $r=0$ & $r=1$ & $r=0$ & $r=1$ & $r=0$ & $r=1$ \\
\hline $2 \times 2$ & 10 & 8 & 12 & 10 & 14 & 12 & 16 & 15 & 19 & 17 & 20 & 20 \\
\hline $4 \times 4$ & - & - & 15 & 16 & 21 & 17 & 29 & 23 & 39 & 33 & 53 & 43 \\
\hline $8 \times 8$ & - & - & - & - & 25 & 21 & 38 & 29 & 54 & 43 & 74 & 61 \\
\hline $16 \times 16$ & - & - & - & - & - & - & 48 & 38 & 73 & 55 & 106 & 82 \\
\hline $32 \times 32$ & - & - & - & - & - & - & - & - & 96 & 75 & 196 & 113 \\
\hline $64 \times 64$ & - & - & - & - & - & - & - & - & - & - & 263 & 206 \\
\hline \multicolumn{13}{|c|}{ OAS(2) - Greville collocation points } \\
\hline & \multicolumn{2}{|c|}{$1 / h=8$} & \multicolumn{2}{|c|}{$1 / h=16$} & \multicolumn{2}{|c|}{$1 / h=32$} & \multicolumn{2}{|c|}{$1 / h=64$} & \multicolumn{2}{|c|}{$1 / h=128$} & \multicolumn{2}{|c|}{$1 / h=256$} \\
\hline$N$ & $r=0$ & $r=1$ & $r=0$ & $r=1$ & $r=0$ & $r=1$ & $r=0$ & $r=1$ & $r=0$ & $r=1$ & $r=0$ & $r=1$ \\
\hline $2 \times 2$ & 11 & 12 & 10 & 11 & 10 & 10 & 11 & 11 & 11 & 11 & 10 & 12 \\
\hline $4 \times 4$ & - & - & 13 & 14 & 14 & 13 & 17 & 15 & 20 & 18 & 24 & 21 \\
\hline $8 \times 8$ & - & - & - & - & 12 & 14 & 13 & 12 & 16 & 14 & 20 & 17 \\
\hline $16 \times 16$ & - & - & - & - & - & - & 12 & 15 & 11 & 11 & 14 & 12 \\
\hline $32 \times 32$ & - & - & - & - & - & - & - & - & 10 & 14 & 9 & 10 \\
\hline $64 \times 64$ & - & - & - & - & - & - & - & - & - & - & 9 & 12 \\
\hline
\end{tabular}

Table 3: Test 2, 2D scalability in $N$ and quasi-optimality in $H / h$. OAS preconditioners on the unit square domain, Greville collocation points. GMRES iteration counts varying the number of subdomains $N$, mesh size $1 / h$ and the overlap parameter $r$, for fixed $p=4, k=3, E=1 e+6, \nu=0.3$.

\subsection{Test 4: solver dependence on $p$ and $k$}

We then study the performance of the non-preconditioned (NP) and OAS preconditioned GMRES solvers with respect to the spline polynomial degree $p$. The model problem is solved on both the unit square and quarter-ring domains. We here fix the mesh size $h=1 / 64$ and the number of subdomains $N=4 \times 4$, with overlaps $r=0$ and $r=1$. The GMRES iteration counts varying $p$ from 3 to 7 and with maximal spline regularity $k=p-1$ are reported in Table 6 . The iteration counts of the NP solver increase significantly with $p$, whereas both $\mathrm{OAS}(1)$ and $\mathrm{OAS}(2)$ preconditioners exhibit a robust behavior for increasing degree $p$.

\subsection{Test 5: solver robustness with respect to $2 D$ domain deformation}

In this section, we investigate the dependence of the non-preconditioned (NP) and the OAS preconditioned GMRES solvers on domain deformations. We consider the four physical domains $A, B, C, D$, which are increasingly more curved starting from the rectangular domain $A$ as illustrated in Figure 3. In these tests, the number of subdomains is fixed to $N=4 \times 4$, the overlap index is $r=0$, the mesh size is $h=1 / 64$, the NURBS parameters $p=3, k=2$ while Greville and superconvergent collocation points are considered. Also, we fix the Young modulus $E=1 e+6$ and the Poisson ratio $\nu=0.3$. The results clearly show a more robust behavior of the OAS preconditioners than the NP solver when the domain is deformed, since the GMRES iteration counts reported in Table 7 increase (from domain $A$ to domain $D$ ) by a factor 3.65 for the NP solver, while the iterations increase only by a factor 1.9 for the $\mathrm{OAS}(2)$ preconditioner.

\subsection{Test 6: $3 D$ scalability in $N$}

A 3D scalability test on the unit cube is reported in Table 8 for the non-preconditioned (NP), OAS(1) and OAS(2) preconditioned GMRES solvers with fixed $H / h=4$ and $N$ increasing up to $6 \times 6 \times 6$ subdomains. The problem is discretized by $p=3, k=2$ and the overlap index $r=0$. 


\begin{tabular}{c|cc|cc|cc|cc|cc}
\hline \multicolumn{10}{c}{$p=3, k=2$, quarter-ring domain } \\
\hline \hline \multicolumn{10}{c}{ OAS $(1)$ - Greville collocation points } \\
\hline \multirow{2}{*}{$N$} & \multicolumn{1}{c}{$1 / h=8$} & \multicolumn{2}{c}{$1 / h=16$} & \multicolumn{2}{c}{$1 / h=32$} & \multicolumn{2}{c}{$1 / h=64$} & \multicolumn{2}{c}{$1 / h=128$} \\
& $r=0$ & $r=1$ & $r=0$ & $r=1$ & $r=0$ & $r=1$ & $r=0$ & $r=1$ & $r=0$ & $r=1$ \\
\hline $2 \times 2$ & 12 & 10 & 17 & 12 & 24 & 18 & 33 & 25 & 44 & 34 \\
$4 \times 4$ & - & - & 25 & 16 & 35 & 25 & 48 & 36 & 66 & 49 \\
$8 \times 8$ & - & - & - & - & 50 & 32 & 70 & 50 & 95 & 70 \\
$16 \times 16$ & - & - & - & - & - & - & 96 & 63 & 151 & 95 \\
$32 \times 32$ & - & - & - & - & - & - & - & - & 200 & 124 \\
\hline \hline
\end{tabular}

OAS $(2)$ - Greville collocation points

\begin{tabular}{c|cc|cc|cc|cc|cc}
\hline & \multicolumn{2}{|c|}{$1 / h=8$} & \multicolumn{2}{|c|}{$1 / h=16$} & \multicolumn{2}{c|}{$1 / h=32$} & \multicolumn{2}{c|}{$1 / h=64$} & \multicolumn{2}{c}{$1 / h=128$} \\
$N$ & $r=0$ & $r=1$ & $r=0$ & $r=1$ & $r=0$ & $r=1$ & $r=0$ & $r=1$ & $r=0$ & $r=1$ \\
\hline $2 \times 2$ & 12 & 13 & 16 & 13 & 20 & 17 & 26 & 21 & 34 & 27 \\
$4 \times 4$ & - & - & 18 & 16 & 23 & 18 & 31 & 24 & 39 & 31 \\
$8 \times 8$ & - & - & - & - & 24 & 18 & 31 & 24 & 40 & 32 \\
$16 \times 16$ & - & - & - & - & - & - & 28 & 22 & 37 & 29 \\
$32 \times 32$ & - & - & - & - & - & - & - & - & 29 & 22 \\
\hline \hline
\end{tabular}

OAS(1) - Superconvergent collocation points

\begin{tabular}{c|cc|cc|cc|cc|cc}
\hline & \multicolumn{2}{|c|}{$1 / h=8$} & \multicolumn{2}{|c|}{$1 / h=16$} & \multicolumn{2}{c|}{$1 / h=32$} & \multicolumn{2}{c|}{$1 / h=64$} & \multicolumn{2}{c}{$1 / h=128$} \\
$N$ & $r=0$ & $r=1$ & $r=0$ & $r=1$ & $r=0$ & $r=1$ & $r=0$ & $r=1$ & $r=0$ & $r=1$ \\
\hline $2 \times 2$ & 15 & 12 & 19 & 15 & 25 & 20 & 32 & 26 & 42 & 34 \\
$4 \times 4$ & - & - & 27 & 20 & 38 & 27 & 49 & 38 & 64 & 50 \\
$8 \times 8$ & - & - & - & - & 53 & 39 & 72 & 53 & 92 & 70 \\
$16 \times 16$ & - & - & - & - & - & - & 100 & 73 & 138 & 97 \\
$32 \times 32$ & - & - & - & - & - & - & - & - & 190 & 138 \\
\hline \hline
\end{tabular}

OAS(2) - Superconvergent collocation points

\begin{tabular}{c|cc|cc|cc|cc|cc}
\hline & \multicolumn{2}{|c|}{$1 / h=8$} & \multicolumn{2}{c|}{$1 / h=16$} & \multicolumn{2}{c|}{$1 / h=32$} & \multicolumn{2}{c|}{$1 / h=64$} & \multicolumn{2}{c}{$1 / h=128$} \\
$N$ & $r=0$ & $r=1$ & $r=0$ & $r=1$ & $r=0$ & $r=1$ & $r=0$ & $r=1$ & $r=0$ & $r=1$ \\
\hline $2 \times 2$ & 15 & 14 & 17 & 15 & 21 & 18 & 26 & 22 & 32 & 27 \\
$4 \times 4$ & - & - & 20 & 17 & 25 & 21 & 31 & 26 & 39 & 32 \\
$8 \times 8$ & - & - & - & - & 25 & 21 & 31 & 27 & 39 & 32 \\
$16 \times 16$ & - & - & - & - & - & - & 30 & 24 & 36 & 29 \\
$32 \times 32$ & - & - & - & - & - & - & - & - & 31 & 24 \\
\hline
\end{tabular}

Table 4: Test 2, 2D scalability in $N$ and quasi-optimality in $H / h$. OAS preconditioners on the quarter-ring domain, Greville and Superconvergent collocation points. GMRES iteration counts varying the number of subdomains $N$, mesh size $1 / h$ and overlap parameter $r$, for fixed $p=3, k=2, E=1 e+6, \nu=0.3$. 


\begin{tabular}{l|c|c|c|c|c|c}
\hline \multicolumn{6}{c}{ Quarter-ring domain, Greville collocation points } \\
\hline$\nu$ & \multicolumn{3}{|c|}{$p=3, k=2$} & \multicolumn{3}{c}{$p=4, k=3$} \\
& $\mathrm{NP}$ & $\mathrm{OAS}(1)$ & $\mathrm{OAS}(2)$ & $\mathrm{NP}$ & OAS $(1)$ & $\mathrm{OAS}(2)$ \\
\hline 0.30 & 149 & 35 & 23 & 169 & 28 & 21 \\
0.40 & 169 & 38 & 28 & 178 & 31 & 23 \\
0.49 & 376 & 67 & 46 & 384 & 45 & 34 \\
0.499 & 1284 & 127 & 69 & 1752 & 74 & 51 \\
0.4999 & 2254 & 190 & 80 & 16130 & 161 & 88 \\
\hline
\end{tabular}

Table 5: Test 3, OAS performance when $\nu \rightarrow 0.5$ for $E=1 e+6$. Iteration counts of non-preconditioned (NP) GMRES, 1-level $(\mathrm{OAS}(1))$ and 2-level $(\mathrm{OAS}(2))$ preconditioned GMRES with Greville collocation points. Fixed $1 / h=32, N=4 \times 4, H / h=8$.

\begin{tabular}{c|c|cc|cc|c|cc|cc}
\hline \multicolumn{9}{c}{$h=1 / 64, N=4 \times 4(H / h=16)$, Greville collocation points } \\
\hline \hline$p$ & \multicolumn{3}{c}{ unit square domain } & \multicolumn{4}{c}{ quarter-ring domain } \\
& NP & \multicolumn{2}{c}{ OAS $(1)$} & \multicolumn{2}{c}{ OAS $(2)$} & NP & \multicolumn{2}{c}{ OAS $(1)$} & \multicolumn{2}{c}{ OAS $(2)$} \\
& & $r=0$ & $r=1$ & $r=0$ & $r=1$ & & $r=0$ & $r=1$ & $r=0$ & $r=1$ \\
\hline 3 & 220 & 40 & 29 & 22 & 18 & 289 & 48 & 36 & 31 & 24 \\
4 & 249 & 32 & 25 & 18 & 16 & 349 & 40 & 31 & 26 & 22 \\
5 & 274 & 36 & 27 & 19 & 16 & 417 & 45 & 35 & 27 & 22 \\
6 & 376 & 30 & 24 & 17 & 15 & 496 & 39 & 31 & 24 & 21 \\
7 & 443 & 33 & 25 & 17 & 15 & 561 & 45 & 34 & 25 & 20 \\
\hline
\end{tabular}

Table 6: Test 4, solver dependence on $p$ and $k$. GMRES iteration counts for increasing polynomial degree $p$ and the regularity $k=p-1$. Non-preconditioned (NP) GMRES, 1-level and 2-level OAS preconditioners with Greville collocation points on the unit square and quarter-ring domain. Fixed $1 / h=64, N=4 \times 4, H / h=16$, overlaps $r=0$ and $r=1$.

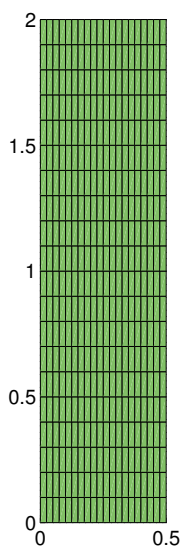

(a)

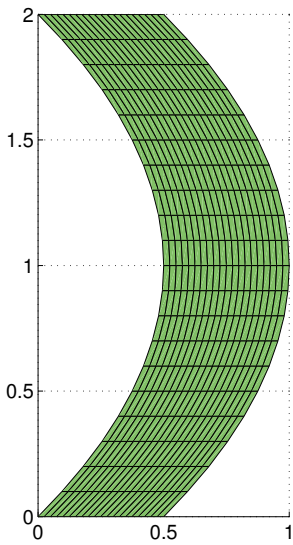

(b)

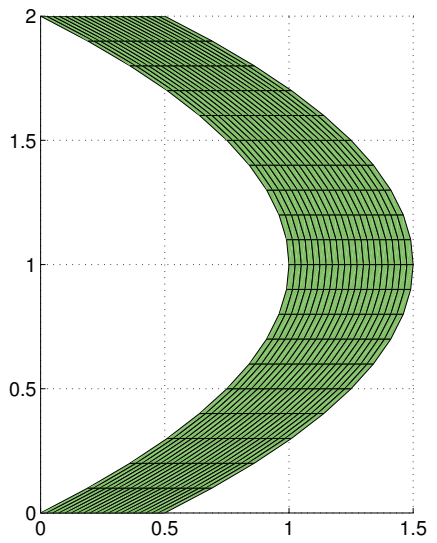

(c)

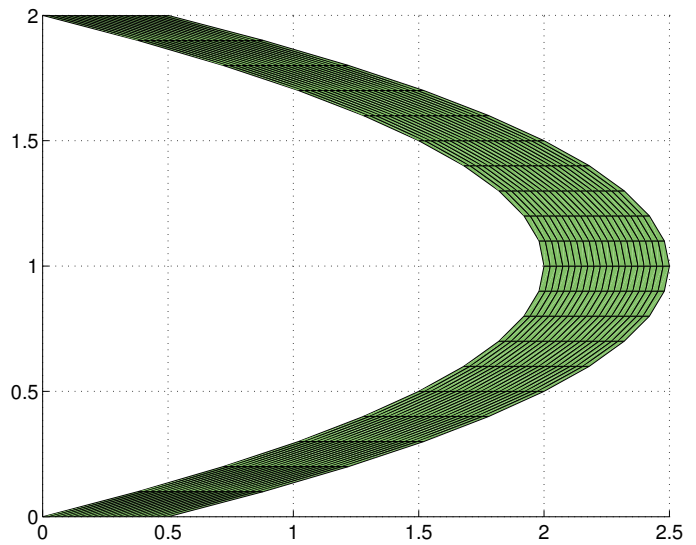

(d)

Figure 3: Test 5, domains for the 2D boomerang test. 


\begin{tabular}{c|c|c|c|c|c|c}
\hline & \multicolumn{3}{|c|}{ Greville collocation points } & \multicolumn{3}{c}{ Superconvergent collocation points } \\
domain & NP & OAS $(1)$ & OAS $(2)$ & NP & OAS $(1)$ & OAS $(2)$ \\
\hline A & 649 & 36 & 24 & 854 & 47 & 24 \\
B & 932 & 54 & 33 & 1321 & 55 & 34 \\
C & 1426 & 55 & 38 & 1669 & 57 & 39 \\
D & 2397 & 57 & 46 & 3117 & 58 & 46 \\
\hline
\end{tabular}

Table 7: Test 5, sovler robustness with respect to 2D domain deformations. Iteration counts of non-preconditioned (NP) GMRES, OAS(1) and OAS(2) preconditioned GMRES with Greville and superconvergent collocation points. Fixed parameters $h=1 / 64, N=4 \times 4, H / h=16, p=3, k=2, r=0, E=1 e+6, \nu=0.3$.

\begin{tabular}{|c|c|c|c|c|c|c|c|c|c|c|c|}
\hline \multicolumn{12}{|c|}{ Cube domain, $H / h=4$, Greville collocation points } \\
\hline \multirow[t]{3}{*}{ 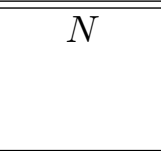 } & \multirow[t]{3}{*}{ 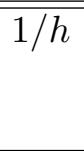 } & \multicolumn{5}{|c|}{$p=2, k=1$} & \multicolumn{5}{|c|}{$\bar{p} p=3, k=2$} \\
\hline & & \multirow[t]{2}{*}{ NP } & \multicolumn{2}{|c|}{$\mathrm{OAS}(1)$} & \multicolumn{2}{|c|}{$\mathrm{OAS}(2)$} & \multirow[t]{2}{*}{ NP } & \multicolumn{2}{|c|}{$\mathrm{OAS}(1)$} & \multicolumn{2}{|c|}{$\mathrm{OAS}(2)$} \\
\hline & & & $r=0$ & $r=1$ & $r=0$ & $r=1$ & & $r=0$ & $r=1$ & $r=0$ & $r=1$ \\
\hline $2 \times 2 \times 2$ & 8 & 26 & 15 & 12 & 16 & 14 & 34 & 18 & 13 & 14 & 16 \\
\hline $3 \times 3 \times 3$ & 12 & 39 & 20 & 19 & 17 & 20 & 49 & 25 & 16 & 17 & 17 \\
\hline $4 \times 4 \times 4$ & 16 & 52 & 24 & 20 & 16 & 19 & 64 & 31 & 19 & 17 & 17 \\
\hline $5 \times 5 \times 5$ & 20 & 65 & 29 & 22 & 16 & 19 & 80 & 38 & 22 & 17 & 17 \\
\hline $6 \times 6 \times 6$ & 24 & 77 & 34 & 24 & 16 & 18 & 96 & 44 & 26 & 17 & 17 \\
\hline
\end{tabular}

Table 8: Test 6, 3D scalability with respect to the number of subdomains $N$. Iteration counts of non-preconditioned (NP) GMRES, OAS(1) and OAS(2) preconditioners. Greville collocation points and parameters $H / h=4, E=1 e+6$, $\nu=0.3$.

As a consequence, both $h$ and $H$ are decreasing proportionally as in a weak scaling test. Only the $\mathrm{OAS}(2)$ preconditioner exhibits a scalable behavior, because the GMRES iteration counts remain bounded from above independently of $N$, while NP and OAS(1) have iteration counts increasing with $N$.

Finally, we study the spectrum of the collocation stiffness matrix and OAS preconditioned operators, reported in Figure 4 for a 3D test with $N=2 \times 2 \times 2$ subdomains and $h=1 / 8$ (top panel) and $N=4 \times 4 \times 4$ subdomains and $h=1 / 16$ (bottom panel). In the top row in each panel, we see the increasing ill-conditioning of the collocation stiffness matrix, since the eigenvalues grow unbounded in absolute value with increasing $N$. The plot in the bottom row in each panel show instead the scalability of the $\mathrm{OAS}(2)$ preconditioner, since the eigenvalues of the preconditioned system remain confined in a box bounded away from the origin of the complex plane, a condition which is often associated to fast GMRES convergence (see e.g. [51]). On the contrary, the plots in the middle row in each panel confirm the non-scalability of $\operatorname{OAS}(1)$, since the eigenvalues of the OAS(1) preconditioned system approach the complex plane origin as $N$ increases.

\section{Conclusions}

Overlapping Additive Schwarz (OAS) preconditioners for collocation IGA approximations of linear elasticity equations have been constructed and studied numerically in both two and three spatial dimensions. The proposed preconditioners relies on a geometric decomposition of the computational domain into overlapping subdomains and solving independent local collocation problems on the overlapping subdomains, thus obtaining a one-level OAS(1) preconditioner. The two-level 
$2 \times 2 \times 2$ subdomains, $h=1 / 8$

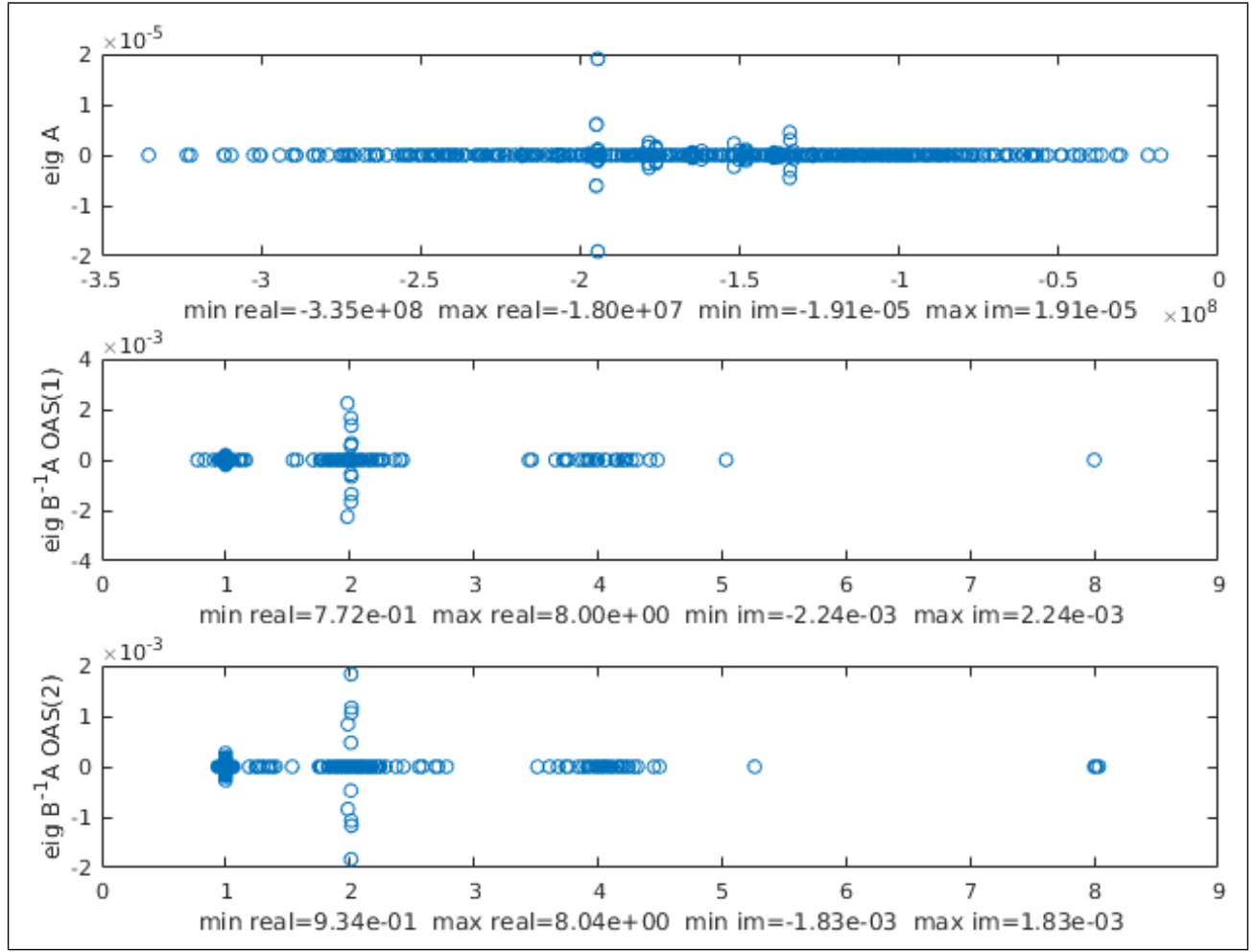

$4 \times 4 \times 4$ subdomains, $h=1 / 16$
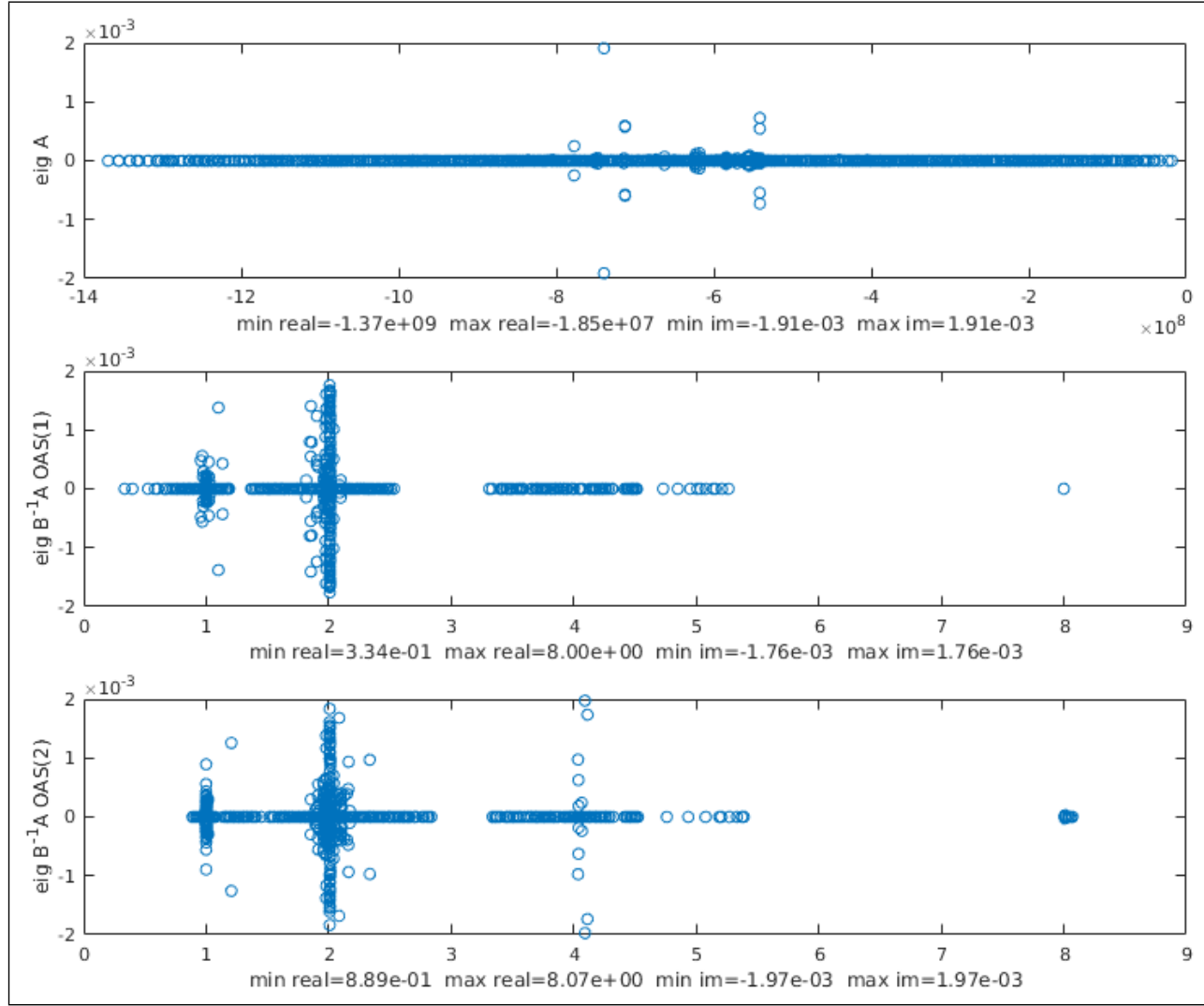

Figure 4: Complex eigenvalues (spectrum) of the collocation unpreconditioned and preconditioned operators. Top panel: $2 \times 2 \times 2$ subdomains, $h=1 / 8$. Bottom panel: $4 \times 4 \times 4$ subdomains, $h=1 / 16$. In each panel: spectrum of the collocation stiffness matrix (first row), one-level OAS(1) preconditioned operator (second row), two-level OAS(2) preconditioned operator (third row). 
preconditioner $(\mathrm{OAS}(2))$ is obtained by augmenting $\mathrm{OAS}(1)$ with an proper collocation coarse problem based on the coarse subdomain mesh. The resulting discrete linear system arising from the collocation IGA approximation is non-symmetric, hence the OAS preconditioners are accelerated by the GMRES iterative solver. The results of our numerical study have shown the OAS $(2)$ quasi-optimality with respect to the ratio $H / h$ and its scalability with respect to the number of subdomains $N$. We have also investigated the behavior of the solvers in terms of the polynomial degree $p$ and regularity index $k$, showing that, differently from what happens when the OAS solvers are applied to the IGA Galerkin approach [9], the iteration counts are very robust with respect to these parameters, even for minimal overlap. Moreover, both OAS solvers are more robust than the non-preconditioned GMRES solver when the material approaches the incompressibility limit $(\nu \approx 0.5)$ and with respect to geometric domain deformations.

\section{Acknowledgments}

Luca F. Pavarino and S. Scacchi would like to acknowledge INDAM-GNCS for the support. D. Cho was partially supported by Basic Science Research Program through the National Research Foundation of Korea (NRF) funded by the Ministry of Education (2018R1D1A1B07048773). This support is gratefully acknowledged.

\section{References}

[1] F. Auricchio, L. Beirão da Veiga, T.J.R. Hughes, A. Reali and G. Sangalli. Isogeometric Collocation Methods. Math. Mod. Meth. Appl. Sci., 20 (11): 2075-2107, 2010.

[2] F. Auricchio, L. Beirão da Veiga, T.J.R. Hughes, A. Reali and G. Sangalli. Isogeometric collocation for elastostatics and explicit dynamics. Comput. Meth. Appl. Mech. Eng., 249-252: $2-14,2012$.

[3] F. Auricchio, L. Beirão da Veiga, J. Kiendl, C. Lovadina and A. Reali. Locking-free isogeometric collocation methods for spatial Timoshenko rods. Comput. Meth. Appl. Mech. Eng., 263: 113$126,2013$.

[4] F. Auricchio, F. Calabro, T.J.R. Hughes, A. Reali and G. Sangalli. A simple algorithm for obtaining nearly optimal quadrature rules for NURBS-based isogeometric analysis. Comput. Meth. Appl. Mech. Eng., 249-252: 15-27, 2012.

[5] Y. Bazilevs, L. Beirão da Veiga, J.A. Cottrell, T.J.R. Hughes and G. Sangalli. Isogeometric analysis: approximation, stability and error estimates for $h$-refined meshes. Math. Mod. Meth. Appl. Sci., 16: 1-60, 2006.

[6] Y. Bazilevs, V. M. Calo, T. J. R. Hughes and Y. Zhang. Isogeometric fluid-structure interaction: theory, algorithms, and computations. Comput. Mech., 43 (1): 3-37, 2008.

[7] L. Beirão da Veiga, A. Buffa, G. Sangalli and R. Vazquez. Mathematical analysis of variational isogeometric methods. ACTA Numerica, 23: 157-287, 2014.

[8] L. Beirão da Veiga, D. Cho, L.F. Pavarino and S. Scacchi. Overlapping Schwarz methods for Isogeometric Analysis. SIAM J. Numer. Anal., 50: 1394-1416, 2012.

[9] L. Beirão da Veiga, D. Cho, L.F. Pavarino and S. Scacchi. Isogeometric Schwarz preconditioners for linear elasticity systems. Comput. Meth. Appl. Mech. Eng., 253: 439-454, 2013. 
[10] L. Beirão da Veiga, D. Cho, L.F. Pavarino and S. Scacchi. BDDC preconditioners for Isogeometric Analysis. Math. Mod. Meth. Appl. Sci., 23: 1099-1142, 2013.

[11] L. Beirão da Veiga, D. Cho, L.F. Pavarino and S. Scacchi. Overlapping Schwarz preconditioners for isogeometric collocation methods. Comput. Meth. Appl. Mech. Eng., 278: 239-253, 2014.

[12] L. Beirão da Veiga, C. Lovadina and A. Reali. Avoiding shear locking for the Timoshenko beam problem via isogeometric collocation methods. Comp. Meth. Appl. Mech. Engrg., 241244:38-51, 2012.

[13] L. Beirão da Veiga, L.F. Pavarino, S. Scacchi, Olof B. Widlund, S. Zampini. Isogeometric BDDC preconditioners with deluxe scaling. SIAM J. Sci. Comput., 36: A1118-A1139, 2014.

[14] L. Beirão da Veiga, L.F. Pavarino, S. Scacchi, Olof B. Widlund, S. Zampini. Adaptive selection of primal constraints for isogeometric BDDC deluxe preconditioners. SIAM J. Sci. Comput., 39: A281-A302, 2017.

[15] A. Buffa, H. Harbrecht, A. Kunoth and G. Sangalli. BPX-preconditioning for isogeometric analysis. Comp. Meth. Appl. Mech. Engrg., 265:63-70, 2013.

[16] A. Buffa, G. Sangalli and R. Vázquez. Isogeometric analysis in electromagnetics: B-splines approximation. Comp. Meth. Appl. Mech. Engrg., 199 (17-20): 1143-1152, 2010.

[17] L. A. Charawi. Isogeometric overlapping Schwarz preconditioners for the Bidomain reactiondiffusion system. Comp. Meth. Appl. Mech. Engrg., 319: 472-490, 2017.

[18] D. Cho. Optimal multilevel preconditioners for isogeometric collocation methods. Math. Comput. Sim., to appear, 2019.

[19] D. Cho and R. Vázquez, BPX preconditioners for isogeometric analysis using analysis-suitable T-splines, IMA J. Numer. Anal. (in press), dry032, https://doi.org/10.1093/imanum/dry032

[20] N. Collier, D. Pardo, L. Dalcin, M. Paszynski, V.M. Calo. The cost of continuity: a study of the performance of isogeometric finite elements using direct solvers. Comp. Meth. Appl. Mech. Engrg., 213-216:353-361, 2012.

[21] N. Collier, L. Dalcin, D. Pardo and V.M. Calo. The cost of continuity: Performance of iterative solvers on isogeometric finite elements. SIAM J. Sci. Comput., 35, 767-784, 2013.

[22] J.A. Cottrell, T.J.R. Hughes, Y. Bazilevs. Isogeometric Analysis. Towards integration of CAD and FEA. Wiley, 2009.

[23] C. De Falco, A. Reali, R. Vazquez. GeoPDEs: A research tool for Isogeometric Analysis of PDEs. Adv. Engrg. Softw., 42, 1020-1034, 2011. 2010.

[24] C. de Boor. A practical guide to splines. Springer, 2001.

[25] S. Demko. On the existence of interpolation projectors onto spline spaces, J. of Approx. Theory, 43, 151-156, 1985.

[26] M. Dryja, O. B. Widlund. Domain Decomposition Algorithms with Small Overlap, SIAM J. Sci.Comp., 15(3), 604-620, 1994. 
[27] G.E. Farin. NURBS curves and surfaces: from projective geometry to practical use. A.K. Peters, 1995.

[28] H. Gomez, T. J. R. Hughes, X. Nogueira and V.M. Calo. Isogeometric analysis of the isothermal Navier Stokes Korteweg equations. Comput. Meth. Appl. Mech. Engrg., 199 (25-28): 18281840, 2010.

[29] T.J.R. Hughes, J.A. Cottrell, Y. Bazilevs. Isogeometric analysis: CAD, finite elements, NURBS, exact geometry, and mesh refinement. Comp. Meth. Appl. Mech. Engrg., 194, 41354195, 2005.

[30] T.J.R. Hughes, A. Reali, G. Sangalli. Efficient Quadrature for NURBS-based Isogeometric Analysis. Comp. Meth. Appl. Mech. Engrg., 199, 301-313, 2010.

[31] M. Donatelli, C. Garoni, C. Manni, S. Serra-Capizzano and H. Speleers. Symbol-based multigrid methods for Galerkin B-spline isogeometric analysis. SIAM J. Numer. Anal., 55 (1): $31-62,2017$.

[32] K. Gahalaut, J. Kraus and S. Tomar. Multigrid Methods for Isogeometric Discretization. Comput. Meth. Appl. Mech. Engrg., 253: 413-425, 2013.

[33] P. Gervasio, L. Dedé, O. Channon and A. Quarteroni. Comparing isogeometric analysis and spectral element methods: accuracy and spectral properties. MOX report 21/2018, Politecnico di Milano, 2018.

[34] H. Gomez, A. Reali and G. Sangalli. Accurate, efficient, and (iso)geometrically flexible collocation methods for phase-field models. J. Comput. Phys., 262: 153-171, 2014.

[35] C. Hofer. Analysis of discontinuous Galerkin dual-primal isogeometric tearing and interconnecting methods. Math. Mod. Meth. Appl. Sci., 28 (1): 131-158, 2018.

[36] C. Hofer. Parallelization of continuous and discontinuous Galerkin dual-primal isogeometric tearing and interconnecting methods. Comput. Math. Appl., 74 (7): 1607--1625, 2017.

[37] C. Hofer and U. Langer. Dual-primal isogeometric tearing and interconnecting solvers for multipatch dG-IgA equations. Comput. Methods Appl. Mech. Eng., 316: 2-21, 2017.

[38] C. Hofer, U. Langer and I. Toulopoulos. Isogeometric analysis on non-matching segmentation: discontinuous Galerkin techniques and efficient solvers. J. Appl. Math. Comput., 61: 297-336, 2019 .

[39] C. Hofreither and S. Takacs. Robust multigrid for isogeometric analysis based on stable splittings of spline spaces. SIAM J. Numer. Anal. 55 (4): 2004-2024, 2017.

[40] S. K. Kleiss, C. Pechstein, B. Jüttler and S. Tomar. IETI-Isogeometric Tearing and Interconnecting. Comput. Meth. Appl. Mech. Eng., 247-248: 201-215, 2012.

[41] M. Montardini, G. Sangalli and L. Tamellini. Optimal-order isogeometric collocation at Galerkin superconvergent points, Comput. Methods Appl. Mech. Engrg., 316: 741-757, 2017.

[42] M. Montardini, G. Sangalli and M. Tani. Robust isogeometric preconditioners for the Stokes system based on the Fast Diagonalization method. Comput. Meth. Appl. Mech. Eng., 338: 162-185, 2018. 
[43] L.F. Pavarino, S. Scacchi, O.B. Widlund and S. Zampini. Isogeometric BDDC deluxe preconditioners for linear elasticity. Math. Mod. Meth. Appl. Sci., 28 (7): 1337-1370, 2018.

[44] L. Piegl and W. Tiller. The NURBS Book, 2nd Edition. Springer-Verlag, 1997.

[45] D.F. Rogers. An Introduction to NURBS With Historical Perspective. Academic Press, 2001.

[46] D. Schillinger, J. A. Evans, A Reali, M. A. Scott, T. J.R. Hughes. Isogeometric Collocation: cost comparison with Galerkin methods and extension to adaptive hierarchical NURBS discretizations. Comp. Meth. Appl. Mech. Engrg., 267:170-232, 2013.

[47] B. F. Smith, P. Bjørstad, W. D. Gropp, Domain Decomposition: Parallel Multilevel Methods for Elliptic Partial Differential Equations, Cambridge University Press, 1996.

[48] G. Sangalli and M. Tani. Isogeometric preconditioners based on fast solvers for the Sylvester equation. SIAM J. Sci. Comput., 38 (6): A3644-A3671, 2016.

[49] M. Tani. A preconditioning strategy for linear systems arising from nonsymmetric schemes in isogeometric analysis. Comput. Math. Appl., 74 (7): 1690-1702, 2017.

[50] A. Toselli, O. B. Widlund. Domain Decomposition Methods: Algorithms and Theory. Computational Mathematics, Vol. 34. Springer-Verlag, Berlin, 2004.

[51] L. N. Trefethen, D. Bau. Numerical Linear Algebra. SIAM, 1996.

[52] R. Vázquez. A new design for the implementation of isogeometric analysis in Octave and Matlab: GeoPDEs 3.0. Comput. Math. Appl., 72 (3): 523-554, 2016.

[53] E. Zampieri and L. F. Pavarino. Explicit second order isogeometric discretizations for acoustic wave problems. Comput. Meth. Appl. Mech. Eng., 348: 776-795, 2019. 